\title{
Violência sobre a pessoa idosa: um estudo documental
}

\author{
Violence against the elderly: a documentary study
}

Miriam Fernanda Sanches Alarcon ${ }^{1}$, Daniela Garcia Damaceno ${ }^{1}$, Carlos Alberto Lazarini ${ }^{2}$, Luzmarina Aparecida Doretto Braccialli², Viviane Boacnin Yoneda Sponchiado ${ }^{3}$, Maria José Sanches Marin ${ }^{2}$

Objetivo: associar as características sociodemográficas de idosos vítimas de violência e dos agressores com os tipos de violência. Métodos: trata-se de um estudo transversal, analítico, retrospectivo, desenvolvido em uma Delegacia. Foram analisados 346 boletins de ocorrência, sendo que as análises estatísticas relativas às comparações foram realizadas com o software Statistical Package for Social Sciences versão 25.0.0.0. Resultados: observou-se associação positiva entre região da cidade, sexo, estado conjugal, escolaridade, idade, local de ocorrência, embasamento legal e forma de notificação da vítima com o tipo de violência, com predomínio da violência financeira em homens e das mulheres nos outros tipos, de cor de pele branca, com companheiro, no domicílio da vítima. Houve predomínio de agressores do sexo masculino. Conclusão: os boletins de ocorrência indicaram a associação de diferentes dados sociodemográficos da vítima e do agressor com os tipos de violência. Descritores: Idoso; Envelhecimento; Violência; Saúde do Idoso; Direitos dos Idosos.

Objective: to associate the sociodemographic characteristics of elderly victims of violence and of the aggressors with types of violence. Methods: this is a cross-sectional, analytical, retrospective study developed in a police station. A total of 346 police reports were analyzed, and the statistical analyses of comparisons were made in the Statistical Package for the Social Sciences version 25.0.0.0. Results: type of violence was positively associated with area of the city, sex, marital status, schooling, age, place of occurrence, legal basis, and form of notification of the victim. There was a predominance of financial violence among men, while other types of violence, white skin, presence of a partner, and the victim's home as the place of occurrence predominated among women. There was a predominance of male aggressors. Conclusion: the police reports indicated the association of different sociodemographic data of victims and aggressors with the types of violence.

Descriptors: Aged; Aging; Violence; Health of the Elderly; Aged Rights.

\footnotetext{
${ }^{1}$ Universidade Estadual Paulista "Júlio de Mesquita Filho". Botucatu, SP, Brasil.

${ }^{2}$ Faculdade de Medicina de Marília. Marília, SP, Brasil.

${ }^{3}$ Delegacia de Polícia de Defesa da Mulher de Marília. Marília, SP, Brasil.
} 


\section{Introdução}

O Brasil é um país que apresenta aumento significativo na população de idosos e, consequentemente, está vivenciando o surgimento de necessidades de saúde complexas e duradouras ${ }^{(1)}$. 0 envelhecimento populacional é um fenômeno visto como impactante, uma vez que promove desequilíbrios entre os diversos grupos etários, tornando inconsistentes as políticas e recursos sociais existentes, sendo necessário que a população mais jovem se torne mais consciente da importância de sua participação nos cuidados dos $\operatorname{idosos}^{(1-2)}$.

Para as famílias, torna-se cada vez mais provável a necessidade de conviver com um familiar idoso $^{(3)}$. Essa relação é permeada por diferentes contextos, como a existência de problemas psiquiátricos, uso álcool/drogas, entre outros que levam desequilíbrio às relações intergeracionais e tornam as pessoas idosas vulneráveis aos maus-tratos, sejam estes físicos, psicológicos e/ou financeiros, principalmente quando o idoso apresenta algum grau de dependência para os cuidados $^{(2,4)}$.

As manifestações de violência contra a pessoa idosa podem ser classificadas como física/sexual, psicológica, financeira, negligência e violação dos direitos individuais. 0 abuso físico inclui ações com intenção de causar dor física ou lesão, como empurrar, agarrar, bater e agredir com uma arma ou objeto, enquanto o abuso sexual inclui comportamentos sexuais ofensivos, assim como contato físico de natureza sexual. A violência psicológica está relacionada a ações com intenção de causar dor emocional, angústia e aflição( ${ }^{(5)}$.

A negligência é caracterizada como a recusa ou falha dos responsáveis por não providenciar ao idoso, dependente de cuidados, assistência em tarefas de vida diária, tais como alimentação, vestuário, abrigo, saúde e cuidados médicos, ou, ainda, ao abandono. A exploração financeira/material é descrita como o uso indevido, por pessoas cuidadoras ou familiares, do dinheiro ou bens materiais pertencentes ao idoso ${ }^{(5)}$. De- vido às especificidades de atuação, a atenção primária à saúde mostra-se indispensável no cuidado ao idoso. Dessa forma, os profissionais devem estar preparados para o desenvolvimento de estratégias de assistência e acolhimento a esses idosos ${ }^{(6)}$.

Contudo, percebe-se a falta de preparo dos profissionais de saúde em lidar com a temática, seja pelas dificuldades em perceber a situação ou pela grande exposição desses profissionais no território. Nessa perspectiva, emerge nos serviços de saúde a necessidade de abordar o tema promovendo e prevenindo as situações de violência, instrumentalizando os profissionais para não apenas identificar os sinais de violência, como também as situações de risco às quais os idosos de estão expostos. Assim, em razão do papel do enfermeiro na assistência direta e na supervisão da equipe de enfermagem e de outros profissionais sua atuação mostra-se essencial para a assistência integral ao idoso vítima de violência ${ }^{(6)}$.

Ao associar o contexto apresentado à banalização da violência no cotidiano das relações familiares e nas formas de negligência social, assim como a falta de preparo dos profissionais em lidar com as situações de violência, compreende-se a importância de imergir na temática da violência contra essa população $0^{(7)}$. Nesse sentido, elaborou-se a seguinte questão de pesquisa: Quais as condições sociodemográficas e os tipos de violência a que as pessoas idosas são submetidas em um município do interior paulista?

O presente estudo, portanto, objetivou associar as características sociodemográficas de idosos vítimas de violência e dos agressores com os tipos de violência.

\section{Métodos}

Estudo transversal, analítico, retrospectivo, desenvolvido na Delegacia de Defesa da Mulher, da Central de Polícia Judiciária da Polícia Civil do Estado de São Paulo de um município de médio porte do interior de São Paulo, Brasil, cuja população de idosos representa $13,6 \%{ }^{(8)}$. 
A Central de Polícia Judiciária da Polícia Civil do Estado de São Paulo, do município, está organizada em dez unidades distribuídas em cinco distritos policiais, quatro delegacias especializadas que atendem às demandas do município todo (Delegacia de Homicídios, Delegacia de Polícia Defesa da Mulher, Delegacia de Investigações sobre Entorpecentes e Núcleo Especial Criminal) e uma Delegacia da Polícia Civil de Plantão. Por não haver uma delegacia especializada na assistência ao idoso vítima de violência no município, a Delegacia de Defesa da Mulher atende a todos os casos de abuso contra a pessoa idosa ${ }^{(9)}$.

Foram analisados Boletins de Ocorrência, registrados na delegacia de polícia de defesa da mulher, no período de outubro de 2016 a março de 2017, em um total de 346 ocorrências de violência contra idosos de ambos os sexos. Roteiro de coleta de dados, com informações referentes às características sociodemográficas (sexo, idade, estado civil, escolaridade, cor da pele) e tipo de violência. Caracterizados dados sociodemográficos do agressor, bem como os encaminhamentos/desfechos jurídicos e sociais das denúncias. Boletins disponibilizados aos pesquisadores em uma sala sede da delegacia.

Variáveis categorizadas em dependente, quanto aos tipos de violência (física; física/sexual; psicológica/moral; negligência/abandono; financeira), e independentes, quanto a sexo, idade, cor da pele, estado civil, escolaridade; caracterização do agressor quanto a sexo, idade, cor da pele, profissão, estado civil, escolaridade, relação com a vítima; e relação entre vítima/ agressor, local de ocorrência, embasamento legal e forma de denúncia. É valido ressaltar que houve perdas de dados relacionados à falta de informações nos boletins de ocorrências, principalmente referentes às características do agressor.

Os critérios de inclusão foram as ocorrências de violência sofridas pelo idoso, aqui classificado como pessoa com idade igual ou superior a 60 anos, de ambos os sexos e residente no município da coleta dos dados. Os critérios de exclusão foram não residir no município estudado; denúncias sem dados acerca da idade da vítima; boletins de ocorrência que não se enquadrassem como violência contra a pessoa idosa.

Os dados foram digitados em planilha eletrônica, análises estatísticas foram realizadas com o software Statistical Package for Social Sciences versão 25.0.0.0. Análises Teste de Qui-quadrado de Pearson e a extensão do teste Exato de Fisher. Conclusões obtidas pelas análises inferenciais com nível de significância $\alpha$ igual a $5 \%(\mathrm{p} \leq 0,050)$.

0 projeto foi aprovado pelo comitê de Ética em Pesquisa da Faculdade de Medicina de Marília, Certificado de Apresentação para Apreciação Ética no 73664417.1.0000.5413, respeitando as normas de pesquisa envolvendo os seres humanas na resolução 466/2012 do Conselho Nacional de Saúde, conforme parecer $\mathrm{n}$ - 2.253.887/2017.

\section{Resultados}

Na Tabela 1, observam-se os dados da distribuição do tipo de violência e as variáveis dependentes dos idosos vítimas de violência registrados na delegacia.

Quanto às características socioeconômicas das vítimas, constata-se associação positiva $(\mathrm{p}<0,050)$ entre sexo e os tipos de violência, sendo que os homens apresentam predomínio da violência financeira e as mulheres dos demais tipos. Houve associação positiva entre a escolaridade da vítima e o tipo de violência, sendo que o ensino fundamental predominou em todos os tipos de violência, e entre os não alfabetizados a violência psicológica/moral foi proporcionalmente maior.

A Tabela 2 apresenta distribuição da relação entre o tipo de violência, as variáveis independentes do agressor e a relação agressor/vítima registrados na delegacia. 
Tabela 1 - Distribuição do tipo de violência e as variáveis dependentes das vítimas registrados na delegacia

\begin{tabular}{|c|c|c|c|c|c|c|c|}
\hline \multirow[b]{2}{*}{ Variáveis } & \multicolumn{7}{|c|}{ Tipos de Violência } \\
\hline & Financeira & Física & $\begin{array}{c}\text { Psicológica/ } \\
\text { Moral }\end{array}$ & Sexual & $\begin{array}{l}\text { Violências asso- } \\
\text { ciadas }\end{array}$ & Total & $\mathbf{p}^{*}$ \\
\hline Sexo & & & & & & & $<0,010$ \\
\hline Masculino & 103 & 15 & 35 & 1 & 4 & 158 & \\
\hline Feminino & 90 & 16 & 57 & 3 & 22 & 188 & \\
\hline Idade (anos) & & & & & & & $<0,010$ \\
\hline $60-69$ & 113 & 20 & 62 & 1 & 9 & 205 & \\
\hline $70-79$ & 54 & 10 & 25 & 3 & 9 & 101 & \\
\hline$>80$ & 26 & 1 & 5 & 0 & 8 & 40 & \\
\hline Cor da pele & & & & & & & $<0,050$ \\
\hline Branco & 167 & 25 & 75 & 4 & 16 & 287 & \\
\hline Negro & 3 & 0 & 6 & 0 & 0 & 9 & \\
\hline Pardo & 15 & 5 & 9 & 0 & 4 & 33 & \\
\hline Amarelo & 6 & 0 & 2 & 0 & 2 & 10 & \\
\hline Não informado & 2 & 1 & 0 & 0 & 4 & 7 & \\
\hline Estado conjugal & & & & & & & $<0,010$ \\
\hline Com companheiro & 98 & 18 & 39 & 2 & 7 & 164 & \\
\hline Sem companheiro & 74 & 10 & 46 & 1 & 6 & 137 & \\
\hline Não informado & 21 & 3 & 7 & 1 & 13 & 45 & \\
\hline Escolaridade & & & & & & & $<0,010$ \\
\hline Não alfabetizado & 1 & 0 & 7 & 0 & 1 & 9 & \\
\hline Fundamental & 64 & 11 & 41 & 2 & 4 & 122 & \\
\hline Médio & 36 & 5 & 22 & 1 & 3 & 67 & \\
\hline Superior & 38 & 4 & 8 & 0 & 1 & 51 & \\
\hline Não Informado & 54 & 11 & 14 & 1 & 17 & 97 & \\
\hline Total & 193 & 31 & 92 & 4 & 26 & 346 & \\
\hline
\end{tabular}

*Teste de Qui-quadrado de Pearson e a extensão de teste exato de Fisher 
Tabela 2 - Distribuição da relação entre o tipo de violência, as variáveis independentes do agressor e a relação agressor/vítima registrados na delegacia

\begin{tabular}{|c|c|c|c|c|c|c|c|}
\hline \multirow[b]{2}{*}{ Variáveis } & \multicolumn{7}{|c|}{ Tipos de violência } \\
\hline & Financeira & Física & $\begin{array}{c}\text { Psicológica/ } \\
\text { Moral }\end{array}$ & Sexual & $\begin{array}{c}\text { Violências asso- } \\
\text { ciadas }\end{array}$ & Total & $\mathbf{p}^{*}$ \\
\hline Sexo & & & & & & & $<0,010$ \\
\hline Masculino & 31 & 15 & 63 & 2 & 13 & 124 & \\
\hline Feminino & 13 & 9 & 20 & 1 & 6 & 49 & \\
\hline Não informado & 149 & 7 & 9 & 1 & 7 & 173 & \\
\hline Idade & & & & & & & 0,510 \\
\hline$\geq 17$ & 0 & 1 & 1 & 1 & 0 & 3 & \\
\hline $18-59$ & 11 & 11 & 35 & 1 & 7 & 65 & \\
\hline$>60$ & 1 & 3 & 5 & 0 & 1 & 10 & \\
\hline Não informada & 181 & 16 & 51 & 2 & 18 & 268 & \\
\hline Situação ocupacional & & & & & & & $<0,050$ \\
\hline Empregado & 14 & 4 & 17 & 0 & 2 & 37 & \\
\hline Desempregado & 2 & 2 & 5 & 0 & 0 & 9 & \\
\hline Não informado & 177 & 25 & 70 & 4 & 24 & 300 & \\
\hline Cor da pele & & & & & & & $<0,010$ \\
\hline Branco & 22 & 17 & 51 & 3 & 10 & 103 & \\
\hline Negro & 1 & 1 & 5 & 0 & 0 & 7 & \\
\hline Pardo & 14 & 3 & 19 & 0 & 2 & 38 & \\
\hline Amarelo & 0 & 0 & 1 & 0 & 0 & 1 & \\
\hline Não informado & 156 & 10 & 16 & 1 & 14 & 197 & \\
\hline Escolaridade & & & & & & & $<0,010$ \\
\hline Não alfabetizado & 0 & 0 & 0 & 1 & 0 & 1 & \\
\hline Fundamental & 5 & 4 & 9 & 1 & 0 & 19 & \\
\hline Médio & 3 & 0 & 3 & 0 & 0 & 6 & \\
\hline Superior & 0 & 0 & 3 & 0 & 0 & 3 & \\
\hline Não informado & 185 & 27 & 77 & 2 & 26 & 317 & \\
\hline Relações agressor/vítima & & & & & & & $<0,010$ \\
\hline Familiar & 7 & 14 & 41 & 1 & 17 & 80 & \\
\hline Profissional & 1 & 0 & 2 & 0 & 0 & 3 & \\
\hline Não familiar & 13 & 8 & 33 & 0 & 2 & 56 & \\
\hline Desconhecida & 172 & 9 & 16 & 3 & 7 & 207 & \\
\hline Total & 193 & 31 & 92 & 4 & 26 & 346 & \\
\hline
\end{tabular}


Constata-se associação positiva $(\mathrm{p}<0,010)$ e predomínio do sexo masculino, empregado, cor autodeclarada branca e com ensino fundamental na violência psicológica/moral. Contudo, percebe-se que as informações dos agressores se encontram incompletas nos boletins de ocorrência ou não foram coletadas. Sobre a relação agressor/vítima, os agressores desconhecidos são aqueles com tendência a praticarem violência financeira e os familiares, violência psicológica/moral.

Na Tabela 3 encontram-se as distribuições dos tipos de violência registrados na delegacia em relação ao local de ocorrência, embasamento legal e forma de denúncia.

Tabela 3 - Distribuição dos tipos de violência registrados na delegacia

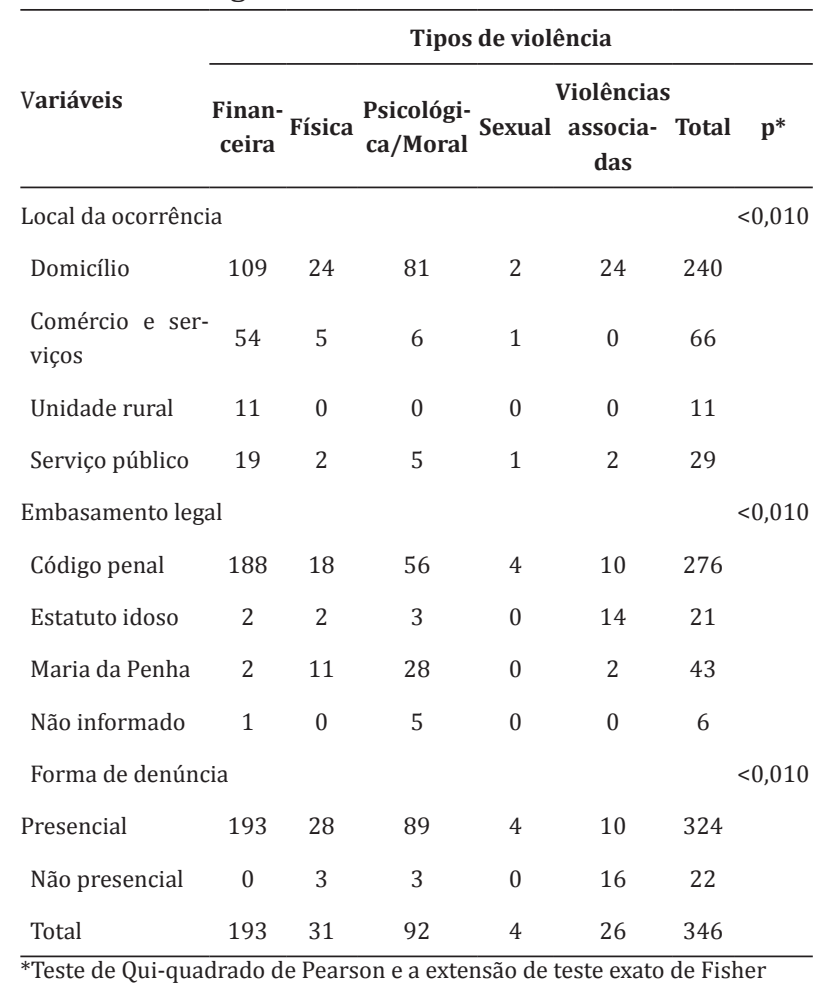

As agressões ocorreram predominantemente no domicílio da vítima, com embasamento legal no código penal, de forma presencial, associando-se positivamente $(p<0,05)$ com violência financeira, sendo esta a mais denunciada.

\section{Discussão}

A falta de informação nos registros e ocorrências foi o principal fator limitante deste estudo, o que dificultou especialmente a caracterização dos agressores, além de ter sido realizado em único município. Assim, faz-se necessário o registro adequado das denúncias de maus-tratos contra idosos, especialmente no que se refere ao agressor, a fim de contribuir com informações para que profissionais das áreas envolvidas na assistência aos mesmos (social, jurídica e saúde) possam intervir de forma mais efetiva.

Entretanto, por meio dos dados apresentados, é possível verificar a dimensão do problema e sua complexidade. A violência financeira, o tipo de violência mais encontrado entre as ocorrências analisadas, leva o idoso a sofrer consequências, como baixa autoestima, tristeza e depressão, entre outros problemas de saúde, sendo necessário que os profissionais da saúde estejam preparados para identificar esse tipo de situação, com vistas a propor intervenções ${ }^{(10)}$. Os fatores de risco de violência contra o idoso demonstraram que os atos de violência ocorrem devido a problemas cognitivos, fragilidade, necessidades relacionadas à saúde física e viuvez do idoso. Além disso, é comum estresse do cuidador e transmissão geracional de comportamentos violentos ${ }^{(11)}$.

Segundo a literatura, 78,9\% de pessoas idosas vítimas de violência são do sexo feminino ${ }^{(12)}$, semelhante ao que se constata no presente estudo. Fato que pode ser explicado pela representação das mulheres para a sociedade, sendo marcadas por preconceitos e represálias em diversos âmbitos e diversas faixas etárias, agravando-se na velhice ${ }^{(13)}$.

Em relação à idade da vítima, os dados do presente estudo vêm ao encontro de achados que mostram predominância da faixa etária 60-65 anos, indicando a relação da independência e autonomia dos idosos facilitando o processo de denúncia ${ }^{(14)}$. Idosos mais velhos encontram, muitas vezes, dificuldades em procurar o órgão especializado. Assim, devido aos obstáculos no acesso e aos receios e medos que envol- 
vem o seu cotidiano deixam, algumas vezes, de realizar as denúncias ${ }^{(5)}$.

Acrescenta-se que o medo de sofrer abandono ou retaliação por seus familiares, de ser levado a lares para idosos, assim como a baixa autoestima pelo seu estado de dependência física e econômica e até mesmo os sentimentos contraditórios que envolvem a denúncia de pessoas próximas, prejudicando-os legalmente, aumentam a probabilidade desses idosos omitirem situações de abuso $^{(12,15)}$.

Estudo realizado no Nordeste do país confirma que idosos que viviam acompanhados sofriam mais violência do que aqueles que viviam só(7). Nesta pesquisa também houve maior prevalência daqueles que vivem com companheiros, exceto quando se trata da violência psicológica/moral.

A escolaridade dos idosos também está relacionada com a capacidade de compreender o abuso e fazer algo para reduzir ou até mesmo evitá-lo. Nas áreas rurais, é possível que por contarem com baixa escolaridade, aumenta-se a chance de idosos sofrerem violência ${ }^{(16)}$. Assim, a baixa escolaridade e a falta de companhia formam um conjunto de fatores de riscos para situações que levam aos maus-tratos da pessoa idosa, especialmente a negligência ${ }^{(17)}$.

Estudo que apresenta a caracterização sociodemográfica dos agressores evidencia que a maioria é do sexo masculino, possui relação familiar com a vítima, principalmente filho $(66,4 \%)$, e com idade média de 46 anos de idade ${ }^{(12)}$, semelhante ao que foi encontrado no presente trabalho, embora a maioria deles não tenha sido identificada.

Segundo relatório do Wall Street Jornal, houve um aumento significativo de jovens desempregados buscando voltar a seus antigos lares, morando, novamente, com pais ou demais parentes já idosos ${ }^{(10)}$, elevando, possivelmente, os casos de abusos contra pessoa idosa ${ }^{(11)}$.

O idoso, pela aposentadoria ou pela própria condição de diminuição da capacidade funcional, tende a permanecer a maior parte do tempo no domicílio, assim sendo os resultados mostram que este foi o lo- cal de maior número de ocorrências. Isso corrobora achados que mostraram que $84,6 \%$ das ocorrências de violência contra a pessoa idosa ocorreram no próprio domicílio e 9,6\% em estabelecimentos comerciais ${ }^{(12)}$.

Ressalta-se, também, que as mudanças do próprio processo de senescência tornam esses idosos vulneráveis a sofrerem violência em locais públicos por pessoas desconhecidas. Compreendendo a questão do declínio cognitivo, é possível perceber que muitos idosos podem ser facilmente persuadidos por vendedores de má fé, sendo vulneráveis a golpes financeiros, visto que estes possuem técnicas, as quais influenciam psicologicamente a vítima, confundindo o processo cognitivo dos idosos ao tomar uma decisão ${ }^{(18)}$.

Nesse contexto, é possível que o idoso seja facilmente exposto também a intervenções de pessoas que, em um primeiro momento, possam se colocar como fonte de algum benefício, especialmente no sentido emocional ou que se aproveitando da fragilidade física e cognitiva exerça com facilidade esse tipo de violência. Embora em menor proporção, a ocorrência de violência contra o idoso em estabelecimentos comerciais também chama a atenção e reforça a vulnerabilidade do idoso perante as inúmeras ofertas de empréstimos ou outras formas de financiamentos que são realizados em consignação à aposentadoria.

Cabe ao profissional dar procedimento à situação de atendimento à vítima de agressão, sempre buscando criar uma atmosfera de confiança e tranquilidade, frisando o fato de sempre respeitar a vontade da pessoa idosa, porém levando sempre em consideração a capacidade cognitiva/mental dessa pessoa, realizando os encaminhamentos necessários para melhorar a qualidade da assistência à vítima de violência ${ }^{(19)}$.

Conforme o artigo 19, da lei no 10.741 , os profissionais da saúde devem notificar os casos de violência contra o idoso quando confirmarem ou suspeitarem de sua ocorrência. Além de prestar o cuidado à saúde, o idoso deve ser encaminhado para o atendimento em outros setores, como aqueles de atenção jurídica e/ ou social, com vistas à responsabilização do agressor e amenizar seu sofrimento ${ }^{(6)}$. 
Os desafios na efetividade na assistência e no suporte ao idoso vítima de violência podem estar associados à sobrecarga de trabalho, à falta de segurança dos profissionais e à dinâmica de trabalho desarticulada com a rede de proteção, além da deficiência na formação desses profissionais acerca dessa temática. Assim, faz-se necessária a reorganização das redes de atenção e dos cursos formadores desses profissionais considerando a importância dessa problemática.

\section{Conclusão}

Observou-se, na presente investigação, que na maioria dos casos as vítimas de agressões em domicílio possuem o ensino fundamental completo, há predomínio da violência financeira em homens, psicológica/moral em mulheres e vítimas com companheiros em todos os tipos de violência, com exceção da violência psicológica e moral. Quanto ao agressor, houve predomínio do sexo masculino e os agressores desconhecidos são aqueles com tendência a praticarem violência financeira, enquanto os familiares, violência psicológica/moral. Os casos foram notificados de forma presencial, estando embasados pelo código penal indicando que existe a subutilização dos crimes elencados pelo estatuto do idoso. Observa-se, também, a falta de informações acerca dos agressores presente nos boletins de ocorrência, dificultando a caracterização de seus perfis.

\section{Agradecimentos}

À Fundação de Amparo à Pesquisa do Estado de São Paulo, processo no 2017/17562-2, e à Coordenação de Aperfeiçoamento de Pessoal de Nível Superior pelo fomento a esta pesquisa.

\section{Colaborações}

Alarcon MFS, Damaceno DG e Marin MJS contribuíram para concepção, elaboração do projeto, análise, interpretação dos dados e redação do artigo.
Lazarini CA colaborou com análise e interpretação dos dados. Braccialli LAD e Sponchiado VBY colaboraram com revisão crítica relevante do conteúdo intelectual e aprovação final da versão a ser publicada.

\section{Referências}

1. Miranda GMD, Mendes ACG, Silva ALA. Population aging in Brazil: current and future social challenges and consequences. Rev Bras Geriatr Gerontol. 2016; 19(3):507-19. doi: http://dx.doi. org/10.1590/1809-98232016019.150140

2. Martins R, Neto MJ, Andrade A, Albuquerque C. Abuse and maltreatment in the elderly. Atenç Prim. 2014; 46(5):206-9. doi: http://dx.doi. org/10.1016/S0212-6567(14)70093-9

3. Pedro WJA, Mena-Chalco JP. 0 envelhecimento na Sociologia brasileira contemporânea: notas preliminares. Rev Kairós [Internet]. 2015 [citado 2019 jun 13]; 18:31-47. Disponível em: https://www. researchgate.net/publication/300185183_O_envelhecimento_na_Sociologia_brasileira_contemporanea_notas_preliminares

4. Couto AM, Castro EAB, Caldas CP. Experiences to be a family caregiver of dependent elderly in the home environment. Rev Rene. 2016; 17(1):7685.doi:http://dx.doi.org/10.15253/21756783.2016000100011

5. Brownell P. A reflection on gender issues in elder abuse research: Brazil and Portugal. Ciênc Saúde Coletiva.2016; 21(11):3323-30.doi: http://dx.doi. org/10.1590/1413-812320152111.23142016

6. Oliveira KSM, Carvalho FPB, Oliveira LC, Simpson CA, Silva FTL, Martins AGC. Violence against the elderly: the conceptions of nursing professionals regarding detection and prevention. Rev Gaúcha Enferm. 2018; 39:e57462. doi: http://dx.doi. org/10.1590/1983-1447.2018.57462

7. Nogueira CF, Freitas MC, Almeida PC. Violência contra idosos no município de Fortaleza, CE: uma análise documental. Rev Bras Geriatr Gerontol. 2011; 14(3):543-54. doi: http://dx.doi. org/10.1590/S1809-98232011000300014

8. Instituto Brasileiro de Geografia e Estatística. Censo demográfico [Internet]. 2010 [citado 2019 jun. 10]. Disponível em: https://cidades.ibge.gov.br/ brasil/sp/marilia/panorama 
9. Plassa BO, Alarcon MFS, Damaceno DG, Sponchiado VBY, Braccialli LAD, Silva JAVE, et al. Flowchart of elderly care victms of abuse: an interdisciplinary perspective. Esc Ana Nery. 2018; 22(4):e20180021. doi: http://dx.doi.org/10.1590/2177-9465-ean-2018-0021

10. Sampaio TSO, Sousa WP, Sampaio LS, Ferreira MJS, Prado APS. Violência financeira em idosos. C\&D - Rev Eletr FAINOR [Internet]. 2017 [citado 2019 mar. 5]; 10(3):363-75. Disponível em: http://srv02.fainor.com.br/revista/index.php/ memorias/article/view/665

11. Gil AP, Santos AJ, Nicolau R, Santos C. Fatores de risco de violência contra as pessoas idosas: consensos e controvérsias em estudos de prevalência. Configurações. 2015; 16:75-95. doi: http://dx.doi. org/10.4000/configuracoes.2852

12. Irigaray TQ, Esteves CS, Pacheco JTB, Grassi-Oliveira $R$, Argimon IIL. Maus-tratos contra idosos em Porto Alegre, Rio Grande do Sul: um estudo documental. Estud Psicol. 2016; 33(3):543-51. doi: dx.doi.org/10.1590/1982-02752016000300017

13. Silva ACLG, Coelho EBS, Moretti-Pires RO. O que se sabe sobre o homem autor de violência contra a parceira íntima: uma revisão sistemática. Rev Panam Salud Pública [Internet]. 2014 [citado 2019 mar. 5]; 35(4):278-83. Disponível em: https:// apps.who.int/iris/handle/10665/295893
14. Garbin CAS, Joaquim RC, Rovida TAS, Garbin AJI. Elderly victims of abuse: a five year document analysis. Rev Bras Geriatr Gerontol. 2016; 19(1):87-94. doi: dx.doi.org/10.1590/1809-9823.2016.15037

15. Castro VC, Rissardo LK, Carreira L.Violence against the Brazilian elderlies: an analysis of hospitalizations. Rev Bras Enferm. 2018; 71(suppl 2):77785. doi: http://dx.doi.org/10.1590/0034-71672017-0139

16. Tonezer C, Trzcinski C, Dal Magro MLP. As vulnerabilidades da velhice rural: um estudo de casos múltiplos no Rio Grande do Sul. Desenvolv Quest. 2017; 15(40):7-38. doi: https://doi. org/10.21527/2237-6453.2017.40.7-38

17. Skirbekk V, James KS. Abuse against elderly in India - the role of education. BMC Public Health. 2014; 14:336. doi: http://dx.doi.org/10.1186/14712458-14-336

18. DeLiema M, YonY, Wilber KH. Tricks of the trade: motivating sales agents to con older adults. Gerontologist. 2016; 56(2):335-44. doi: https://doi. org/10.1093/geront/gnu039

19. Camacho ACLF, Alves RR. Mistreatment against the elderly in the nursing perspective: an integrative review. Rev Enferm UFPE on line [Internet]. 2015 [cited Jun 13, 2019]; 9(2):927-35. Available from:https://periodicos.ufpe.br/revistas / revistaenfermagem/article/view/10418/11201 